\title{
August 10 Highlights
}

\section{Changes in classification of headache disorders}

Lipton et al. summarize the key changes in the recently published second edition of the International Classification of Headache Disorders-2 (ICHD-2). Criteria were developed for several new and treatment responsive forms of headache including the indomethacin-responsive disorders (episodic paroxysmal hemi-crania and hemicrania [continua] and the lithium-responsive disorder, hypnic headache). In addition, chronic migraine, new daily persistent headache, and benign thunderclap headache were added.

see page 427

\section{Early neurologic deterioration in cerebral hemorrhage}

In a prospective study, Leira et al. evaluated predictors and associated factors in 266 intracerebral hemorrhages ( $\mathrm{ICH})$. Clinical and biochemical inflammatory markers were predictors of subsequent early neurologic deterioration, whereas early ICH growth, intraventricular bleeding, and high systolic blood pressure within 48 hours were factors associated with early neurologic deterioration in spontaneous ICH.

see page 461

\section{DVT prevalence and risk factors after brain injury}

Yablon et al. determined the prevalence and risk factors for deep vein thrombosis (DVT) after brain injury. Admission duplex ultrasonography revealed thigh DVT in $11 \%$ of 709 consecutive neurorehabilitation patients. Prevalence was higher with brain tumor (21\%) and hemorrhage (16\%) vs trauma (6.7\%). DVT risk was multifactorial, and influenced by the type of brain injury.

see page 485

\section{IFN $\beta$-1a flu symptoms in MS}

In this double-blind, placebo-controlled trial, Rio et al. compare the efficacy of acetaminophen, ibuprofen, and prednisone in the treatment of flu symptoms induced by intramuscular IFNß-1a in patients with multiple sclerosis. Ibuprofen conferred the best control of symptoms immediately following IFNß1-a administration.

see page 525

\section{CT angiography assessment of carotid stenosis in acute stroke and TIA}
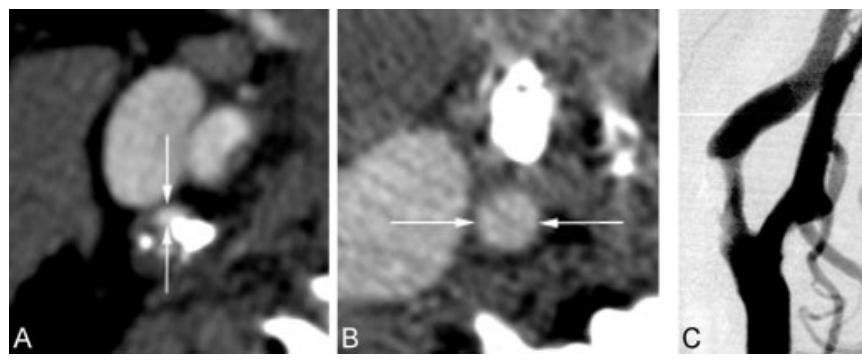

CTA stenosis measurement and example of CTA overcall of stenosis compared with DSA.

Josephson et al. compared CT angiography (CTA) of the neck with conventional angiography (DSA) in a consecutive series of patients (81 vessels). Using a 50 or $70 \%$ stenosis cut-off, CTA had a high negative predictive value (99 to $100 \%$ ), and when coupled with initial CT imaging, can effectively exclude carotid disease.

see page 457

The Josephson et al. article is the second 2004 article on a noninvasive method of assessing carotid stenosis, both accompanied by an editorial. Here, Feasby and Findlay briefly consider three major types of noninvasive carotid imaging: Doppler ultrasound (DUS), contrast enhanced MRI (CEMRA), and CT angiography (CTA). CTA uses widely available CT technology, with the ability to produce anatomic images similar to CEMRA and arteriography (CA). Furthermore, since most stroke and TIA patients are imaged acutely with a head CT, vascular imaging by CTA is easily combined. Perhaps CTA is the ideal candidate to replace CA. Commenting on the Josephson et al. study, they note that while the sensitivity is ideal, the specificity is not. CTA tends to overestimate the degree of stenosis. Having only 5/81 arteries with $>70 \%$ stenosis in their series, they do not have accurate data on the specificity of CTA in high-grade stenosis, the clearest indication for carotid endarterectomy. Moreover, CTA requires IV contrast administration. Before CTA is accepted as the equivalent of arteriography, it needs to be compared with CA in larger series, particularly cases with high-grade stenosis. Emergency CTA evaluation of the carotid bifurcation is not yet an appropriate screening test for acute cerebral ischemia in unselected patients.

see page 412 


\section{Neuropathology of hippocampal sclerosis dementia}

Blass et al. performed a case-control study comparing the clinical course of autopsy-confirmed hippocampal sclerosis $(n=19)$, frontotemporal dementia $(n=24)$, and Alzheimer disease $(n=19)$. Hippocampal sclerosis patients resembled FTD more than $\mathrm{AD}$ patients in the domains of behavior, cognition, and general psychopathology. Most hippocampal sclerosis patients met clinical diagnostic criteria for FTD.

see page 492

Autopsy cases previously diagnosed as hippocampal sclerosis dementia (HSD) were re-evaluated by Hatanpaa et al. Most cases could be reclassified as the motor neuron disease inclusion dementia (MNDID) variant of FTD.

see page 538

In the accompanying editorial Lippa and Dickson note that Blass et al. and Hatanpaa et al. use retrospective data to paint a portrait of HSD as a variant of FTD. These articles consolidate what is known about HSD by providing evidence for a degenerative etiology in many HSD cases. Their data parallel a growing sophistication in the ability to clinically differentiate FTD from $A D$, and they provide evidence that many cases of HSD fall into the broadening spectrum of disorders that would be clinically diagnosed as having features of FTD. However, it is not established that either HSD or FTD has a clear, single etiology.

see page 414

\section{Transcranial sonography discriminates corticobasal degeneration vs progressive supranuclear palsy}

Walter et al. studied 13 patients with PSP and 8 with CBD. Brain parenchyma sonography discriminated CBD from PSP with an 83\% specificity and a 100\% sensitivity. Bilateral substantia nigra hyperechogenicity indicated CBD, whereas third-ventricle dilatation indicated PSP. In this small series, each had a positive predictive value of $100 \%$.

see page 504

\section{Ictal stuttering is a sign of pseudoseizures}

Vossler et al. evaluated 230 adults and found that stuttering during seizures was seen in $8.5 \%$ of patients with psychogenic nonepileptic seizures (PNES) and in none of the patients with epilepsy. PNES cases with stuttering had significantly shorter duration of seizure disorder, stronger conversion profile, and were more likely to be female than epilepsy patients. Ictal stuttering has rarely been reported in childhood epilepsy, but its occurrence in women with recent-onset seizures suggests PNES.

see page 516

\section{Changing lamotrigine kinetics in pregnancy}

De Haan et al. studied lamotrigine kinetics in 12 pregnancies in women with epilepsy on monotherapy. Their data suggest the need for frequent lamotrigine level measurements and dose adjustment during pregnancy to compensate for its doubled clearance.

see page 571

\section{High oxygen flow rates for cluster headache}

Todd Rozen observed that high oxygen flow rates (100\% oxygen via face mask at 14 to $15 \mathrm{~L} / \mathrm{min}$ ) provided cluster headache relief in three patients who had shown no response to standard oxygen cluster headache therapy (flow rates of 7 to $10 \mathrm{~L} / \mathrm{min}$ ).

see page 593

\section{Migraine headache in male to female transsexuals}

Pringsheim and Gooren found that male to female transsexuals taking hormonal therapy for feminization have migraine prevalence similar to genetic females and significantly different from genetic males.

see page 593 


\title{
Neurology
}

\author{
August 10 Highlights \\ Neurology 2004;63;408-409 \\ DOI 10.1212/01.WNL.0000137377.73496.EE
}

This information is current as of August 9, 2004

\section{Updated Information \&}

Services

Permissions \& Licensing

Reprints including high resolution figures, can be found at: http://n.neurology.org/content/63/3/408.full

Information about reproducing this article in parts (figures,tables) or in its entirety can be found online at:

http://www.neurology.org/about/about_the_journal\#permissions

Information about ordering reprints can be found online:

http://n.neurology.org/subscribers/advertise

Neurology ${ }^{\circledR}$ is the official journal of the American Academy of Neurology. Published continuously since 1951, it is now a weekly with 48 issues per year. Copyright. All rights reserved. Print ISSN: 0028-3878. Online ISSN: 1526-632X.

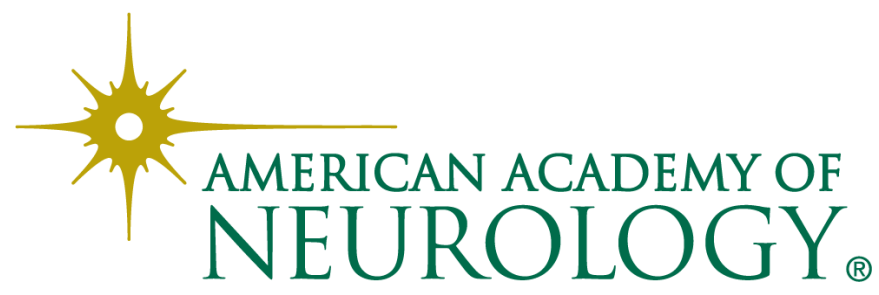

